\title{
Nature of the metallization transition in solid hydrogen
}

\author{
Sam Azadi* \\ Department of Physics, Imperial College London, Thomas Young Centre and London Centre for Nanotechnology, \\ London SW7 2AZ, United Kingdom \\ N. D. Drummond \\ Department of Physics, Lancaster University, Lancaster LA1 4YB, United Kingdom \\ W. M. C. Foulkes \\ Department of Physics, Imperial College London, Exhibition Road, London SW7 2AZ, United Kingdom
}

(Received 31 May 2016; revised manuscript received 3 November 2016; published 24 January 2017)

\begin{abstract}
We present an accurate study of the static-nucleus electronic energy band gap of solid molecular hydrogen at high pressure. The excitonic and quasiparticle gaps of the $C 2 / c, P c, P b c n$, and $P 6_{3} / m$ structures at pressures of 250,300, and $350 \mathrm{GPa}$ are calculated using the fixed-node diffusion quantum Monte Carlo (DMC) method. The difference between the mean-field and many-body band gaps at the same density is found to be almost independent of system size and can therefore be applied as a scissor correction to the mean-field gap of an infinite system to obtain an estimate of the many-body gap in the thermodynamic limit. By comparing our static-nucleus DMC energy gaps with available experimental results, we demonstrate the important role played by nuclear quantum effects in the electronic structure of solid hydrogen.
\end{abstract}

DOI: 10.1103/PhysRevB.95.035142

\section{INTRODUCTION}

Determining the metallization pressure of solid hydrogen is one of the great challenges of high-pressure physics. Since 1935, when it was predicted that molecular solid hydrogen would become a metallic atomic crystal at $25 \mathrm{GPa}$ [1], compressed hydrogen has been studied intensively. Additional interest arises from the possible existence of room-temperature superconductivity [2], a metallic liquid ground state [3], and the relevance of solid hydrogen to astrophysics $[4,5]$.

Early spectroscopic measurements at low temperature suggested the existence of three solid-hydrogen phases [4]. Phase I, which is stable up to $110 \mathrm{GPa}$, is a molecular solid composed of quantum rotors arranged in a hexagonal close-packed structure. Changes in the low-frequency regions of the Raman and infrared spectra imply the existence of phase II, also known as the broken-symmetry phase, above $110 \mathrm{GPa}$. The appearance of phase III at $150 \mathrm{GPa}$ is accompanied by a large discontinuity in the Raman spectrum and a strong rise in the spectral weight of molecular vibrons. Phase IV, characterized by the two vibrons in its Raman spectrum, was recently discovered at $300 \mathrm{~K}$ and pressures above $230 \mathrm{GPa}$ [6-8]. Another new phase has been claimed to exist at pressures above $200 \mathrm{GPa}$ and higher temperatures (for example, $480 \mathrm{~K}$ at $255 \mathrm{GPa}$ ) [9]. This phase is thought to meet phases I and IV at a triple point, near which hydrogen retains its molecular character. The most recent experimental results [10] indicate that $\mathrm{H}_{2}$ and hydrogen deuteride at $300 \mathrm{~K}$ and pressures greater than $325 \mathrm{GPa}$ transform to a new phase $\mathrm{V}$, characterized by substantial weakening of the vibrational Raman activity. Other features include a change in the pressure dependence of the fundamental vibrational frequency and the partial loss of the low-frequency excitations.

\footnotetext{
*s.azadi@imperial.ac.uk
}

Although it is very difficult to reach the hydrostatic pressure of more than $400 \mathrm{GPa}$ at which hydrogen is normally expected to metallize, some experimental results have been interpreted as indicating metallization at room temperature below $300 \mathrm{GPa}$ [6]. However, other experiments show no evidence of the optical conductivity expected of a metal at any temperature up to the highest pressures explored [11]. Experimentally, it remains unclear whether or not the molecular phases III and IV are metallic, although it has been suggested that phase $\mathrm{V}$ may be nonmolecular (atomic) [10]. Metallization is believed to occur either via the dissociation of hydrogen molecules and a structural transformation to an atomic metallic phase $[6,12]$ or via band-gap closure within the molecular phases $[13,14]$. In this work we investigate the latter possibility using advanced computational electronic structure methods.

Structures of crystalline materials are normally determined by x-ray or neutron diffraction methods. These techniques are very challenging for low-atomic-number elements such as hydrogen [15]. Fortunately, optical phonon modes disappear, appear, or experience sudden shifts in frequency when the crystal structure changes. It is therefore possible to identify the transitions between phases using optical methods.

The electronic structures of the solid molecular phases have mainly been investigated using computational methods based on density functional theory (DFT) [16-25] and the quasiparticle (QP) approach within the $G W$ approximation $[14,26]$. Although DFT-based methods can be used to search for candidate low-energy crystal structures and to calculate their vibrational properties, the inadequacies of DFT are more apparent in the case of band-gap calculations [27]. To obtain accurate gaps, it is vital to go beyond mean-field-like methods and solve the many-electron Schrödinger equation directly. In this work, we employ the fixed-node diffusion quantum Monte Carlo (DMC) method to calculate excitonic and QP band gaps of cold dense hydrogen as functions of pressure. 
Fixed-node DMC is the most accurate known method for evaluating the total energies of continuum systems of more than a few tens of interacting quantum particles [28-33]. Recently, it has been indicated that DMC can provide an accurate description of the phase diagram of solid molecular hydrogen [34]. Although the DMC method was originally designed to study ground states, it is also capable of providing accurate information about excited states in atoms, molecules, and crystals [35-38]. DMC calculations of excitations in crystals remain challenging because of a $1 / N$ effect: The fractional change in the total energy due to the presence of a one- or two-particle excitation is inversely proportional to the number of electrons in the simulation cell. Since large simulation cells are required to provide an accurate description of the infinite solid, high-precision calculations are necessary.

The main input to any ab initio calculation is the structure of the system under study, which in this case is unknown. Hence there is no option but to use structures predicted by mean-field methods such as DFT. It is now generally accepted that DFT results for high-pressure hydrogen depend on the choice of exchange-correlation functional $[21,22,25]$. This frustrating limitation may be the main cause of the contradictions $[39,40]$ between existing computational results.

In the present work we use the DMC method to carry out a comprehensive study of the pressure dependence of the energy band gap of solid hydrogen at high pressure. The definitive static-nucleus many-body band-gap data we provide can be used to correct results obtained using less accurate methods. The corrections required are approximately independent of lattice vibrations and temperature.

The paper is organized as follows. Section II describes the details of our DFT and quantum Monte Carlo (QMC) calculations. Section III provides the DMC electronic structure results for molecular solid hydrogen structures at 250,300, and $350 \mathrm{GPa}$. The nature of metallization is discussed in Sec. IV. Section V concludes.

\section{COMPUTATIONAL DETAILS}

\section{A. DFT calculations}

We consider the $C 2 / c, P c, P b c n$, and $P 6_{3} / m$ molecular structures of solid hydrogen at pressures of 250, 300, and $350 \mathrm{GPa}$. According to $a b$ initio calculations, the $C 2 / c$ and $P c$ structures are the most favorable candidates for phases III and IV, respectively [17,34]. The $C 2 / c$ and $P c$ crystals have weakly-bonded graphenelike layers [17], while the $P b c n$ structure includes two different layers of graphenelike threemolecule rings with elongated $H_{2}$ molecules and unbound $H_{2}$ molecules $[16,17]$. The $P 6_{3} / m$ structure may also be viewed as layered, but it is not graphenelike: Three quarters of the $\mathrm{H}_{2}$ molecules lie flat in the plane and one quarter lie perpendicular to the plane. The interplane bonding is relatively strong and the centers of the molecules fall on a slightly distorted hexagonal close-packed lattice [16]. The structures were fully relaxed using DFT at fixed pressure, and the relaxed structures were then used in the DMC simulations. Our DFT calculations were carried out within the pseudopotential and plane-wave approach using the QUANTUM ESPRESSO [41] and CASTEP [42] codes. All our DFT calculations used norm-conserving pseudopotentials, a basis set of plane waves with a cutoff of $100 \mathrm{Ry}$, and the Becke-Lee-Yang-Parr (BLYP) generalized gradient approximation exchange-correlation energy functional [43]. Geometry and cell optimizations employed a dense $16 \times 16 \times 16 k$-point mesh. The Broyden-Fletcher-GoldfarbShanno quasi-Newton algorithm was used for cell and geometry optimization, with convergence thresholds on the total energy and forces of $0.01 \mathrm{mRy}$ and $0.1 \mathrm{mRy} / \mathrm{Bohr}$, respectively, to guarantee convergence of the total energy to better than $1 \mathrm{meV} /$ proton and the pressure to better than $0.1 \mathrm{GPa}$. Internal coordinates and lattice parameters of our optimized structures are reported in the Supplemental Material [44].

We used the BLYP functional because the resulting structures give lower DMC energies than structures determined using the Perdew-Burke-Ernzerhof (PBE) functional [34] Therefore, the BLYP geometries are more accurate and hence DMC band gaps obtained using BLYP geometries should be more accurate than DMC band gaps obtained using PBE geometries. Further evidence in support of our approach is provided by Figs. 3, 4, and 5 of Ref. [22] and also Table I of the Supplemental Material of that work. It was shown that the van der Waals density functional (vdW-DF) [45] geometry of the $C 2 / c$ phase is very close to the DMC geometry; in particular, according to Fig. 5 of Ref. [22], of all the functionals studied, vdW-DF gives the smallest error in the molecular bond length relative to DMC calculations. However, the DFT geometries calculated using the vdW-DF and BLYP functionals are almost the same. To investigate further, we have compared the vdW-DF-optimized geometry with the BLYP-optimized geometry for the $P 6_{3} / \mathrm{m}$ phase at $250 \mathrm{GPa}$. We find that the difference between the resulting molecular bond lengths is $0.0007 \AA$. Therefore we conclude that BLYP is an accurate DFT functional for optimizing the geometry of solid hydrogen.

\section{B. QMC calculations}

Our DMC calculations used Slater-Jastrow trial wave functions as implemented in the CASINO QMC code [46]. The single-particle orbitals were obtained from DFT calculations using the plane-wave-based QUANTUM ESPRESSO code [41]. A norm-conserving pseudopotential constructed within DFT using the BLYP exchange-correlation functional was employed [43]. We chose a very large basis-set cutoff of 200 Ry [47]. The plane-wave orbitals were transformed into a blip spline basis [48] before use in our DMC calculations. A time step of 0.01 a.u. was used for the DMC calculations.

The fixed-node DMC method samples the variationally optimal many-electron wave function consistent with an assumed trial nodal surface. [The nodal surface of an $N$-electron wave function $\Psi\left(\boldsymbol{r}_{1}, \boldsymbol{r}_{2}, \ldots, \boldsymbol{r}_{N}\right)$ is the $(3 N-1)$-dimensional surface on which $\Psi$ is zero.] The trial nodal surface is usually defined by means of a trial wave function, and the quality of the nodal surface of the trial wave function affects the quality of the results. This is the only fundamental approximation in the method.

Our fixed-node DMC results were obtained using real trial wave functions constructed at the $\Gamma$ point of the simulation supercell. To study convergence with cell size and correct the finite-size errors we used various supercells, the smallest and largest of which contained 128 and 864 hydrogen 
TABLE I. DMC results for the $C 2 / c$ structure at pressures $P=250,300$, and $350 \mathrm{GPa}$. The columns headed $E_{0}, E^{\prime}, E_{N+1}$, and $E_{N-1}$ list the total DMC energies in Hartrees of the ground state of a supercell containing $N$ electrons, the first singlet excited state of that supercell, the ground state of the supercell after one electron has been added, and the ground state of the supercell after one electron has been removed, respectively. The columns headed $\Delta_{\mathrm{exc}}, \Delta_{\mathrm{qp}}$, and $\delta_{\text {sci }}$ are the values in $\mathrm{eV}$ of the excitonic band gap, the QP band gap, and the scissor correction.

\begin{tabular}{|c|c|c|c|c|c|c|c|}
\hline \multicolumn{8}{|c|}{$\mathbf{P}=250 \mathrm{GPa}$} \\
\hline $\mathrm{N}$ & $E_{0}$ & $E^{\prime}$ & $E_{N+1}$ & $E_{N-1}$ & $\Delta_{\text {exc }}$ & $\Delta_{\mathrm{qp}}$ & $\delta_{\text {sci }}$ \\
\hline 192 & $-102.288(1)$ & $-102.177(2)$ & $-101.888(2)$ & $-102.571(2)$ & $3.02(6)$ & $3.18(6)$ & $1.08(6)$ \\
\hline 432 & $-230.075(2)$ & $-229.947(3)$ & $-229.662(3)$ & $-230.354(3)$ & $3.5(1)$ & $3.6(1)$ & $1.1(1)$ \\
\hline 648 & $-345.278(2)$ & $-345.112(3)$ & $-344.868(3)$ & $-345.524(3)$ & $4.5(1)$ & $4.5(1)$ & $1.2(1)$ \\
\hline \multicolumn{8}{|c|}{$\mathbf{P}=300 \mathrm{GPa}$} \\
\hline 192 & $-100.803(1)$ & $-100.721(2)$ & $-100.381(2)$ & $-101.142(2)$ & $2.23(6)$ & $2.26(6)$ & $0.81(6)$ \\
\hline 432 & $-226.808(1)$ & $-226.713(3)$ & $-226.377(3)$ & $-227.143(3)$ & $2.6(1)$ & $2.6(1)$ & $0.7(1)$ \\
\hline 648 & $-340.400(1)$ & $-340.278(3)$ & $-339.963(3)$ & $-340.707(3)$ & $3.3(1)$ & $3.5(1)$ & $0.7(1)$ \\
\hline \multicolumn{8}{|c|}{$\mathrm{P}=350 \mathrm{GPa}$} \\
\hline 192 & $-99.377(1)$ & $-99.331(2)$ & $-98.934(2)$ & $-99.775(2)$ & $1.25(6)$ & $1.22(6)$ & $0.6(1)$ \\
\hline 432 & $-223.686(2)$ & $-223.611(3)$ & $-223.225(3)$ & $-224.070(3)$ & $2.0(1)$ & $2.1(1)$ & $0.7(1)$ \\
\hline 648 & $-335.419(2)$ & $-335.306(3)$ & $-334.991(3)$ & $-335.736(3)$ & $3.1(1)$ & $3.0(1)$ & $0.8(1)$ \\
\hline
\end{tabular}

atoms. Our Jastrow factor included polynomial one-body electron-nucleus (1b) terms, two-body electron-electron (2b) terms, three-body electron-electron-nucleus (3b) terms, and plane-wave expansions ( $p$ terms) in the electron-electron separations [49]. The $p$ terms build long-ranged correlations into the Jastrow factor and significantly improve the wave function and variational energy. We also employed a backflow (BF) [50] transformation to introduce more correlation into the trial wave function and thus improve the fixed nodal surface. Our BF transformation included electron-electron and electron-proton terms and is given by

$$
\mathbf{X}_{i}\left(\left\{\mathbf{r}_{j}\right\}\right)=\mathbf{r}_{i}+\boldsymbol{\xi}_{i}^{(e-e)}\left(\left\{\mathbf{r}_{j}\right\}\right)+\boldsymbol{\xi}_{i}^{(e-P)}\left(\left\{\mathbf{r}_{j}\right\}\right),
$$

where $\mathbf{X}_{i}\left(\left\{\mathbf{r}_{j}\right\}\right)$, the transformed coordinate of electron $i$, depends on the full configuration of the system $\left\{\mathbf{r}_{j}\right\}$. The vector functions $\boldsymbol{\xi}_{i}^{(e-e)}\left(\left\{\mathbf{r}_{j}\right\}\right)$ and $\boldsymbol{\xi}_{i}^{(e-P)}\left(\left\{\mathbf{r}_{j}\right\}\right)$ are the electron-electron and electron-proton BF displacements of electron $i$, respectively. They are parameterized as

$$
\boldsymbol{\xi}_{i}^{(e-e)}\left(\left\{\mathbf{r}_{j}\right\}\right)=\sum_{j \neq i}^{N_{e}} \alpha_{i j}\left(r_{i j}\right) \mathbf{r}_{i j}
$$

and

$$
\boldsymbol{\xi}_{i}^{(e-P)}\left(\left\{\mathbf{r}_{j}\right\}\right)=\sum_{I}^{N_{P}} \beta_{i I}\left(r_{i I}\right) \mathbf{r}_{i I},
$$

where $\alpha_{i j}\left(r_{i j}\right)$ and $\beta_{i I}\left(r_{i I}\right)$ are polynomial functions of electron-electron and electron-proton distance, respectively, and contain optimizable parameters. All adjustable parameters in the Jastrow factor and BF function were optimized using variance and energy minimization, respectively, at the variational Monte Carlo (VMC) level [51,52].

The QP energy gap is defined as

$$
\Delta_{\mathrm{qp}}=E_{N+1}+E_{N-1}-2 E_{0},
$$

where $E_{0}$ is the ground-state energy of a system of $N$ electrons and $E_{N+1}\left(E_{N-1}\right)$ is the many-body total energy of the system after an electron has been added to (removed from) the system.
Our calculations of $\Delta_{\mathrm{qp}}$ are performed at the $\Gamma$ point of the supercell Brillouin zone, equivalent to a mesh of $k$ points including $\Gamma$ in the primitive Brillouin zone. We calculate a vertical QP energy gap, assuming that the ground- and excited-state structures are the same. The difference between the vertical and adiabatic QP gaps is expected to be small [53]. We used the same Jastrow factors (and, where relevant, BF functions) for $N-,(N+1)$-, and $(N-1)$-electron systems. We create excitonic states by promoting an electron from a valence-band orbital into a conduction-band orbital with the same Bloch wave vector. The excitonic absorption gap is

$$
\Delta_{\text {exc }}=E^{\prime}-E_{0},
$$

where $E^{\prime}$ is the total energy of the excitonic state. Again we work at the $\Gamma$ point of the supercell Brillouin zone. In the ground-state geometry, the singlet excitonic gap is equivalent to the vertical optical absorption gap [53].

To obtain DMC band gaps in the thermodynamic (infinite supercell) limit, we introduce a scissor correction $\delta_{\mathrm{sci}}(N)$, defined as the difference between the DMC and DFT band gaps of a given supercell at a given density: $\delta_{\text {sci }}(N)=\Delta^{\mathrm{DMC}}(N)-$ $\Delta^{\mathrm{DFT}}(N)$, where $\Delta^{\mathrm{DMC}}(N)$ and $\Delta^{\mathrm{DFT}}(N)$ are DMC and DFT band gaps for a simulation cell containing $N$ atoms.

\section{RESULTS}

\section{A. $C 2 / c$ structure}

The $C 2 / c$ structure has 24 atoms in the primitive unit cell. Our DMC simulations employed supercells of $192(2 \times 2 \times$ $2), 432(2 \times 3 \times 3)$, and $648(3 \times 3 \times 3)$ atoms. Table I lists our DMC results for the $C 2 / c$ phase at pressures of 250,300 , and $350 \mathrm{GPa}$. Slater-Jastrow trial wave functions without $\mathrm{BF}$ were used.

The top panel of Fig. 1 shows the ground-state VMC and DMC energies of the $C 2 / c$ structure at $250 \mathrm{GPa}$. Calculations were carried out with five different Slater-Jastrow trial wave functions: The first included only one- and two-body Jastrow correlations $[\mathrm{SJ}(1 \mathrm{~b}+2 \mathrm{~b})]$; the second had one-, two-, and threebody correlations $[\mathrm{SJ}(1 \mathrm{~b}+2 \mathrm{~b}+3 \mathrm{~b})]$; the third had one-, two-, and three-body correlations and $p$ terms $[\mathrm{SJ}(1 \mathrm{~b}+2 \mathrm{~b}+3 \mathrm{~b}+p)]$ 

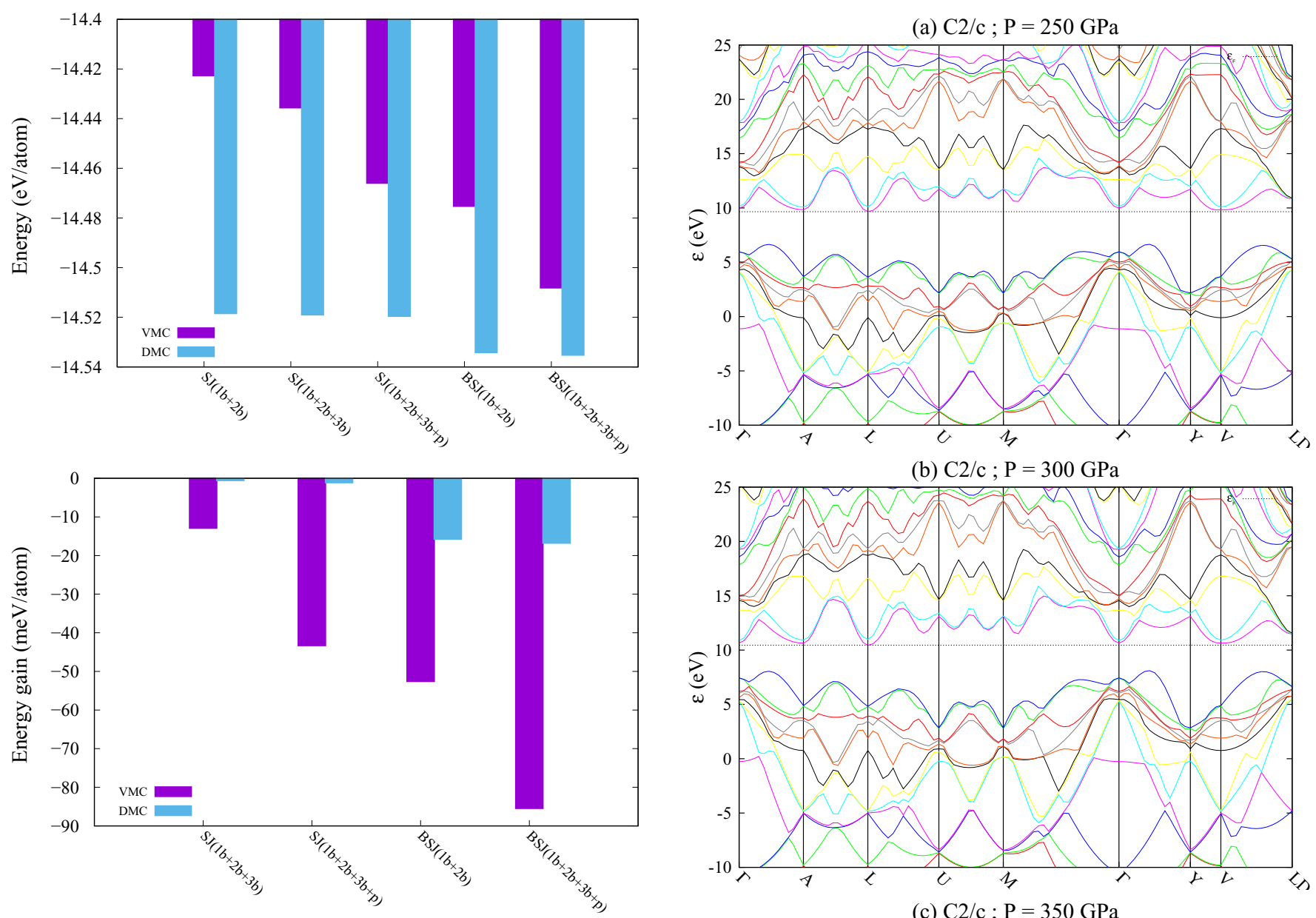

FIG. 1. Top panel: VMC and DMC energies of the $C 2 / c$ structure at $250 \mathrm{GPa}$. Various wave functions were used as explained in the text. Total energies were calculated at the $\Gamma$ point of a simulation cell containing 288 atoms. Bottom panel: VMC and DMC energies relative to their values calculated using a $\mathrm{SJ}(1 b+2 b)$ wave function. The incorporation of BF significantly improves the DMC total energy.

the fourth had one- and two-body correlations and BF $[\mathrm{BSJ}(1 \mathrm{~b}+2 \mathrm{~b})]$; and the fifth had one-, two-, and three-body correlations, $p$ terms, and $\mathrm{BF}[\mathrm{BSJ}(1 \mathrm{~b}+2 \mathrm{~b}+3 \mathrm{~b}+p)]$.

The bottom panel of Fig. 1 shows how the choice of wave function affects the calculated ground-state energy of the $C 2 / c$ structure; energies are relative to the $\mathrm{SJ}(1 b+2 b)$ value, which was chosen as a reference. As expected, adding $3 b$ correlations and $p$ terms to the Jastrow factor reduces the VMC energy substantially, but the DMC energy is not affected because the trial nodal surface is unaltered. Using a BF transformation

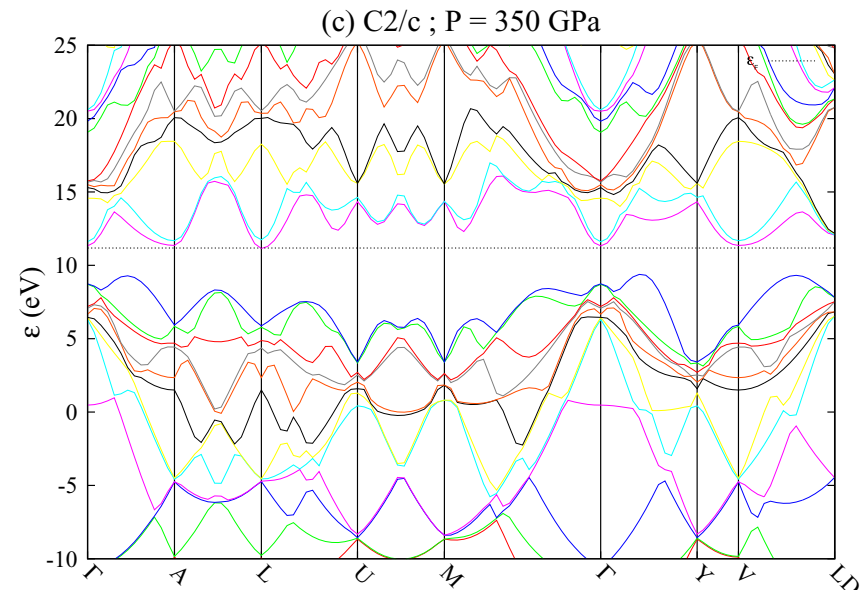

FIG. 2. DMC scissor-corrected DFT-BLYP band structures of the $C 2 / c$ phase at pressures $P=250,300$, and $350 \mathrm{GPa}$. The dashed line shows the Fermi energy.

TABLE II. DMC results for a simulation cell containing $N=192$ atoms in the $C 2 / c$ structure at $250 \mathrm{GPa}$. Calculations were carried out using $\mathrm{SJ}(1 b+2 b)$ and $\mathrm{BSJ}(1 b+2 b)$ wave functions. The columns headed $E_{0}, E_{N+1}$, and $E_{N-1}$ list the ground-state energies in Hartrees of systems containing $N, N+1$, and $N-1$ electrons, respectively. The column headed $E^{\prime}$ lists the energy of the singlet first excited state of the $N$-electron system, again in Hartrees. The columns headed $\Delta_{\text {qp }}$ and $\Delta_{\text {exc }}$ are the QP and excitonic energy gaps in eV.

\begin{tabular}{lcccrr}
\hline \hline Wave fn. & $E_{0}$ & $E^{\prime}$ & $E_{N+1}$ & $E_{N-1}$ & $\Delta_{\text {exc }}$ \\
\hline SJ & $-102.288(1)$ & $-102.177(2)$ & $-101.888(2)$ & $-102.571(2)$ & $3.02(6)$ \\
BSJ & $-102.373(2)$ & $-102.270(4)$ & $-101.986(3)$ & $-102.662(4)$ & $2.18(6)$ \\
\hline \hline
\end{tabular}


TABLE III. DMC results for the $P c$ structure at pressures $P=250,300$, and $350 \mathrm{GPa}$. The columns headed $E_{0}, E^{\prime}, E_{N+1}$, and $E_{N-1}$ list the total DMC energies in Hartrees of the ground state of a supercell containing $N$ electrons, the first singlet excited state of that supercell, the ground state of the supercell after one electron has been added, and the ground state of the supercell after one electron has been removed, respectively. The columns headed $\Delta_{\mathrm{exc}}, \Delta_{\mathrm{qp}}$, and $\delta_{\mathrm{sci}}$ are the values in $\mathrm{eV}$ of the excitonic band gap, the QP band gap, and the scissor correction.

\begin{tabular}{|c|c|c|c|c|c|c|c|}
\hline $\mathrm{N}$ & $E_{0}$ & $E^{\prime}$ & $\mathbf{P}=\underset{E_{N+1}}{\mathbf{2 5 0} \mathbf{~ G P a}}$ & $E_{N-1}$ & $\Delta_{\text {exc }}$ & $\Delta_{\mathrm{qp}}$ & $\delta_{\text {sci }}$ \\
\hline 192 & $-101.410(1)$ & $-101.266(2)$ & $-101.008(2)$ & $-101.672(2)$ & $3.92(8)$ & $3.81(8)$ & $1.79(8)$ \\
\hline 384 & $-203.298(2)$ & $-203.190(3)$ & $-202.885(3)$ & $-203.596(3)$ & $2.9(1)$ & $3.1(1)$ & $1.6(1)$ \\
\hline 576 & $-306.654(2)$ & $-306.502(3)$ & $\begin{array}{r}-306.238(4) \\
\mathbf{P}=\mathbf{3 0 0} \mathbf{~ G P a}\end{array}$ & $-306.926(4)$ & $4.1(1)$ & $3.9(2)$ & $1.7(2)$ \\
\hline$\overline{192}$ & $-99.915(1)$ & $-99.8012(2)$ & $-99.492(2)$ & $-100.214(2)$ & $3.09(8)$ & $3.3(1)$ & $1.7(1)$ \\
\hline 384 & $-200.379(2)$ & $-200.301(3)$ & $-199.951(3)$ & $-200.729(3)$ & $2.1(1)$ & $2.1(1)$ & $1.5(1)$ \\
\hline 576 & $-303.519(2)$ & $-303.395(3)$ & $\begin{array}{r}-303.086(4) \\
\mathbf{P}=\mathbf{3 5 0} \mathbf{~ G P a}\end{array}$ & $-303.834(4)$ & $3.3(1)$ & $3.2(2)$ & $1.6(1)$ \\
\hline 192 & $-98.474(1)$ & $-98.390(2)$ & $-98.024(2)$ & $-98.828(2)$ & $2.28(8)$ & $2.6(1)$ & $1.4(1)$ \\
\hline 384 & $-197.568(2)$ & $-197.522(3)$ & $-197.126(3)$ & $-197.973(3)$ & $1.2(1)$ & $1.0(1)$ & $1.1(1)$ \\
\hline 576 & $-299.483(2)$ & $-299.388(4)$ & $-299.023(4)$ & $-299.853(4)$ & $2.6(1)$ & $2.4(2)$ & $1.3(1)$ \\
\hline
\end{tabular}

improves the trial nodal surface and lowers the calculated energy at both the VMC and DMC levels. Adding BF to a $\mathrm{SJ}(1 b+2 b)$ wave function lowers the DMC energy by $18(1)$ $\mathrm{meV} /$ atom.

The difference between the VMC energies calculated with the $\mathrm{SJ}(1 b+2 b)$ and $\mathrm{SJ}(1 b+2 b+3 b+p)$ wave functions is $45(2) \mathrm{meV} /$ atom, whereas adding $\mathrm{BF}$ correlations to a $\mathrm{SJ}(1 b+2 b)$ wave function lowers the VMC energy by $53(1)$ $\mathrm{meV}$ /atom. The VMC energy gained by introducing $\mathrm{BF}$ is therefore comparable to that gained using $3 b$ correlations and $p$ terms. The difference between the $\mathrm{SJ}(1 b+2 b) \mathrm{DMC}$ energy and $\mathrm{BSJ}(1 b+2 b+3 b+p)$ VMC energy is just $9(2)$ $\mathrm{meV} /$ atom.

Table II lists the DMC QP and excitonic gaps of the $C 2 / c$ structure at $250 \mathrm{GPa}$. Results obtained with Slater-Jastrow (SJ) and BF-Slater-Jastrow (BSJ) wave functions are shown. We used a simulation cell with 192 hydrogen atoms.

Figure 2 illustrates the DMC band structure of the $C 2 / c$ phase at pressures of 250,300 , and $350 \mathrm{GPa}$; the band energies were obtained by adding the DMC-based scissor correction to the DFT-BLYP band structure.

\section{B. $P c$ structure}

The $P c$ structure has 48 atoms in the primitive unit cell. Our DMC simulations employed supercells of $192(2 \times 2 \times 1)$, $384(2 \times 2 \times 2)$, and $576(3 \times 2 \times 2)$ atoms. Table III lists our DMC results for the $P c$ structure at pressures of 250,300 , and $350 \mathrm{GPa}$.

Figure 3 illustrates the DMC band structure of the $P c$ phase at pressures of 250,300 , and $350 \mathrm{GPa}$; the band energies were obtained by adding the DMC-based scissor correction to the DFT-BLYP band structure.

\section{Pbcn structure}

The $P b c n$ structure has 48 atoms in the primitive unit cell. Our DMC simulations employed supercells of $384(2 \times 2 \times$ $2), 576(3 \times 2 \times 2)$, and $864(3 \times 3 \times 2)$ atoms. Table IV lists

TABLE IV. DMC results for the $P b c n$ structure at pressures $P=250,300$, and $350 \mathrm{GPa}$. The columns headed $E_{0}, E^{\prime}, E_{N+1}$, and $E_{N-1}$ list the total DMC energies in Hartrees of the ground state of a supercell containing $N$ electrons, the first singlet excited state of that supercell, the ground state of the supercell after one electron has been added, and the ground state of the supercell after one electron has been removed, respectively. The columns headed $\Delta_{\mathrm{exc}}, \Delta_{\mathrm{qp}}$, and $\delta_{\mathrm{sci}}$ are the values in $\mathrm{eV}$ of the excitonic band gap, the QP band gap, and the scissor correction.

\begin{tabular}{|c|c|c|c|c|c|c|c|}
\hline $\mathrm{N}$ & $E_{0}$ & $E^{\prime}$ & $\mathbf{P}=\underset{E_{N+1}}{250} \mathbf{~ G P a}$ & $E_{N-1}$ & $\Delta_{\mathrm{exc}}$ & $\Delta_{\mathrm{qp}}$ & $\delta_{\text {sci }}$ \\
\hline 384 & $-204.109(2)$ & $-203.969(2)$ & $-203.693(3)$ & $-204.384(3)$ & $3.8(1)$ & $3.8(1)$ & $1.5(1)$ \\
\hline 576 & $-306.573(2)$ & $-306.442(3)$ & $-306.169(3)$ & $-306.845(3)$ & $3.5(1)$ & $3.6(1)$ & $1.4(1)$ \\
\hline \multirow[t]{2}{*}{864} & $-459.801(3)$ & $-459.650(3)$ & $-459.391(4)$ & $-460.052(4)$ & $4.1(1)$ & $4.3(1)$ & $1.4(1)$ \\
\hline & & & $\mathbf{P}=300 \mathrm{GPa}$ & & & & \\
\hline$\overline{384}$ & $-201.177(2)$ & $-201.067(2)$ & $-200.745(3)$ & $-201.499(3)$ & $2.9(1)$ & $3.0(1)$ & $1.4(1)$ \\
\hline 576 & $-302.286(2)$ & $-302.180(3)$ & $-301.857(3)$ & $-302.611(3)$ & $2.9(1)$ & $2.8(1)$ & $1.5(1)$ \\
\hline \multirow[t]{2}{*}{864} & $-453.392(3)$ & $-453.260(3)$ & $-452.942(4)$ & $-453.703(4)$ & $3.6(1)$ & $3.8(2)$ & $1.5(1)$ \\
\hline & & & $\mathrm{P}=350 \mathrm{GPa}$ & & & & \\
\hline 384 & $-198.219(2)$ & $-198.146(2)$ & $-197.764(3)$ & $-198.598(3)$ & $2.0(1)$ & $2.1(1)$ & $0.9(1)$ \\
\hline 576 & $-297.994(2)$ & $-297.927(3)$ & $-297.546(3)$ & $-298.380(3)$ & $1.8(1)$ & $1.7(1)$ & $0.9(1)$ \\
\hline 864 & $-446.970(3)$ & $-446.892(3)$ & $-446.519(4)$ & $-447.334(4)$ & 2.1(1) & $2.3(2)$ & $0.9(1)$ \\
\hline
\end{tabular}




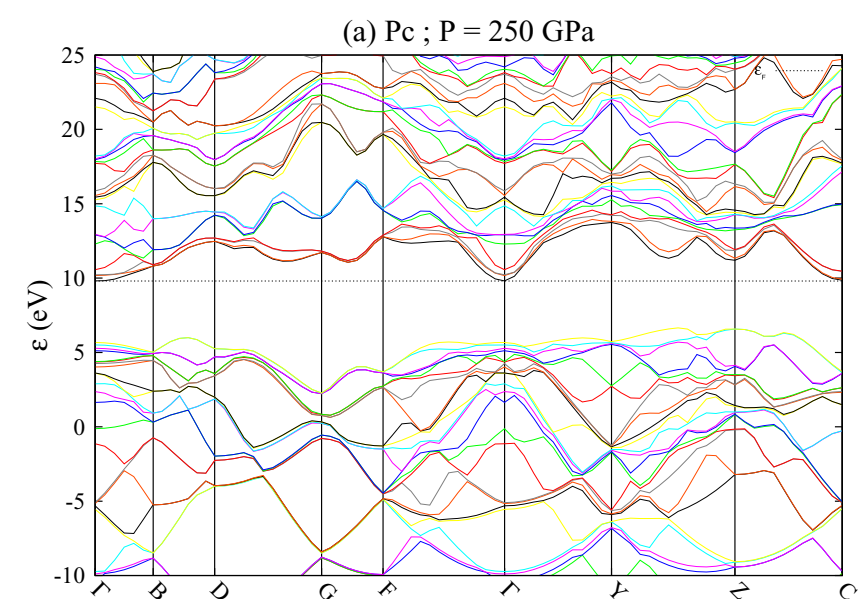

(b) $\mathrm{Pc} ; \mathrm{P}=300 \mathrm{GPa}$

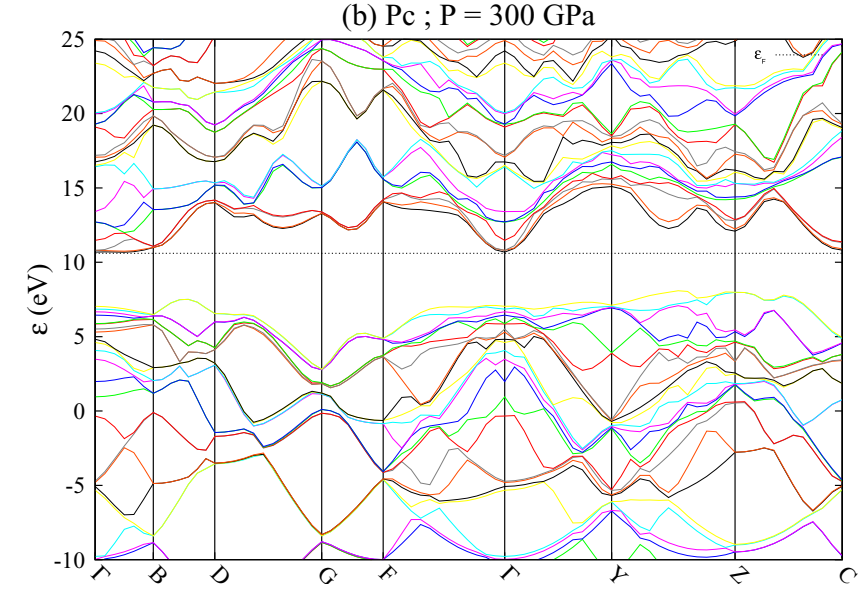

(c) $\mathrm{Pc} ; \mathrm{P}=350 \mathrm{GPa}$

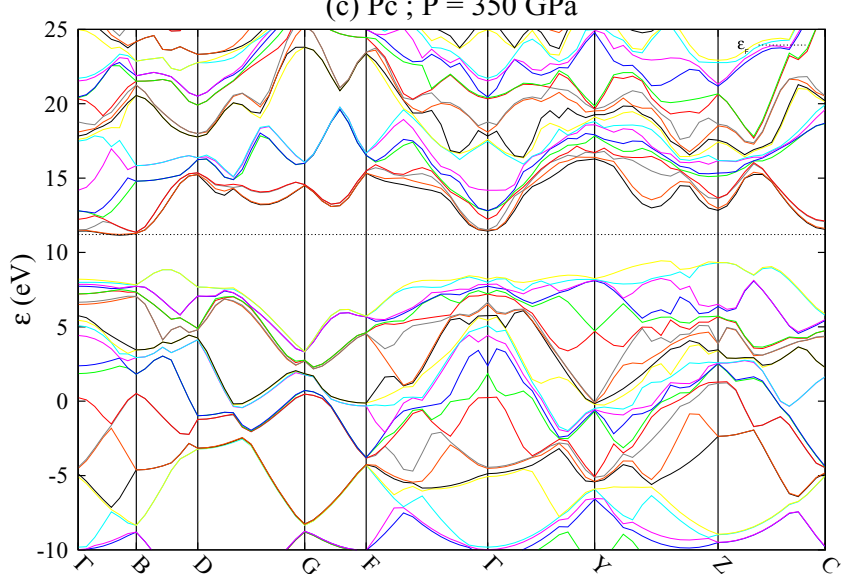

FIG. 3. As Fig. 2, but for the $P c$ phase at pressures $P=250$, 300 , and $350 \mathrm{GPa}$.

our DMC results for the Pbcn phase at pressures of 250, 300, and $350 \mathrm{GPa}$.

Figure 4 illustrates the DMC band structure of the $P b c n$ phase at pressures of 250,300 , and $350 \mathrm{GPa}$; the band energies were obtained by adding the DMC-based scissor correction to the DFT-BLYP band structure.

\section{D. $\mathrm{P6}_{3} / m$ structure}

The $P 6_{3} / m$ structure has 16 atoms in the primitive unit cell. Our DMC simulations employed supercells of 128
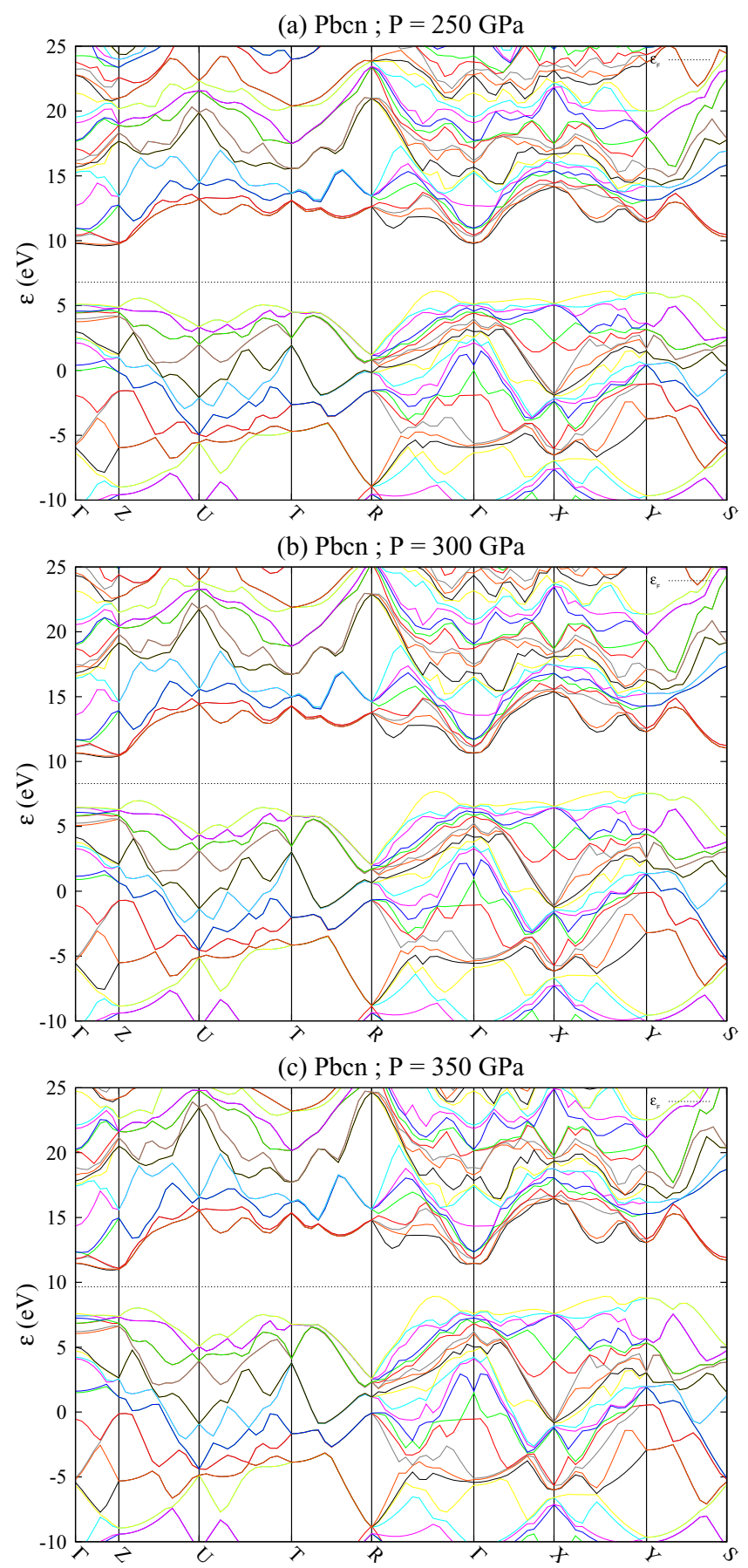

FIG. 4. As Fig. 2, but for the $P b c n$ phase at pressures $P=250$, 300 , and $350 \mathrm{GPa}$.

$(2 \times 2 \times 2), 192(2 \times 2 \times 3), 288(3 \times 2 \times 3)$, and $768(4 \times$ $3 \times 4$ ) atoms. Table $\mathrm{V}$ presents our DMC results for $P 6_{3} / \mathrm{m}$ phase at pressures of 250, 300, and $350 \mathrm{GPa}$.

Figure 5 illustrates the DMC band structure of the $P 6_{3} / \mathrm{m}$ phase at pressures of 250, 300, and $350 \mathrm{GPa}$; the band energies were obtained by adding the DMC-based scissor correction to the DFT-BLYP band structure. 
TABLE V. DMC results for the $P 6_{3} / m$ structure at pressures $P=250,300$, and $350 \mathrm{GPa}$. The columns headed $E_{0}, E^{\prime}, E_{N+1}$, and $E_{N-1}$ list the total DMC energies in Hartrees of the ground state of a supercell containing $N$ electrons, the first singlet excited state of that supercell, the ground state of the supercell after one electron has been added, and the ground state of the supercell after one electron has been removed, respectively. The columns headed $\Delta_{\mathrm{exc}}, \Delta_{\mathrm{qp}}$, and $\delta_{\text {sci }}$ are the values in $\mathrm{eV}$ of the excitonic band gap, the QP band gap, and the scissor correction.

\begin{tabular}{|c|c|c|c|c|c|c|c|}
\hline $\mathrm{N}$ & $E_{0}$ & $E^{\prime}$ & $\mathbf{P}=\underset{E_{N+1}}{\mathbf{2 5 0} \mathbf{~ G P a}}$ & $E_{N-1}$ & $\Delta_{\text {exc }}$ & $\Delta_{\mathrm{qp}}$ & $\delta_{\text {sci }}$ \\
\hline 128 & $-67.887(1)$ & $-67.755(2)$ & $-67.448(2)$ & $-68.197(2)$ & $3.59(6)$ & $3.51(7)$ & $1.2(1)$ \\
\hline 192 & $-102.401(1)$ & $-102.272(2)$ & $-101.956(3)$ & $-102.720(3)$ & $3.51(6)$ & $3.4(1)$ & $1.1(1)$ \\
\hline 288 & $-153.446(2)$ & $-153.303(3)$ & $-152.996(3)$ & $-153.756(3)$ & $3.89(9)$ & $3.8(1)$ & $1.4(1)$ \\
\hline \multirow[t]{2}{*}{768} & $-410.141(3)$ & $-409.998(3)$ & $-409.691(4)$ & $-410.451(4)$ & $3.9(1)$ & $3.8(1)$ & $1.3(1)$ \\
\hline & & & $\mathbf{P}=300 \mathrm{GPa}$ & & & & \\
\hline 128 & $-66.821(1)$ & $-66.718(2)$ & $-66.401(2)$ & $-67.141(2)$ & $2.80(6)$ & $2.7(1)$ & $1.0(1)$ \\
\hline 192 & $-100.918(1)$ & $-100.817(2)$ & $-100.499(3)$ & $-101.237(3)$ & $2.75(6)$ & $2.7(1)$ & $0.9(1)$ \\
\hline 288 & $-151.198(2)$ & $-151.081(3)$ & $-150.757(3)$ & $-151.523(3)$ & $3.2(1)$ & $3.2(1)$ & $1.4(1)$ \\
\hline \multirow[t]{2}{*}{768} & $-403.192(3)$ & $-403.082(3)$ & $-402.757(4)$ & $-403.517(4)$ & $3.0(1)$ & $3.0(1)$ & $1.3(1)$ \\
\hline & & & $P=350 \mathrm{GPa}$ & & & & \\
\hline 128 & $-65.832(1)$ & $-65.760(2)$ & $-65.405(2)$ & $-66.186(2)$ & $1.96(6)$ & $2.0(1)$ & $0.9(1)$ \\
\hline 192 & $-99.501(1)$ & $-99.431(2)$ & $-99.081(3)$ & $-99.850(3)$ & $1.9(1)$ & $1.9(1)$ & $0.7(1)$ \\
\hline 288 & $-149.067(2)$ & $-148.982(3)$ & $-148.647(3)$ & $-149.403(3)$ & $2.3(1)$ & $2.3(1)$ & $1.0(1)$ \\
\hline 768 & $-397.818(3)$ & $-397.735(3)$ & $-397.398(4)$ & $-398.156(4)$ & $2.2(1)$ & $2.2(1)$ & $1.0(1)$ \\
\hline
\end{tabular}

\section{DISCUSSION}

\section{A. Static-nucleus results}

\section{Exciton binding}

We calculated the DMC singlet and triplet excited-state total energies for the $C 2 / c$ phase using a simulation cell containing 192 hydrogen atoms. The singlet excited-state energy was obtained by promoting an electron without flipping its spin; the triplet excited-state energy was obtained by promoting an electron and flipping its spin. The values of the first singlet and triplet excited-state total energies at a pressure of $250 \mathrm{GPa}$ are -102.177 (2) and -102.174(3) Hartrees, respectively; thus the difference in the singlet and triplet excitonic gaps is statistically insignificant. Indeed, we find that the singlet and triplet exciton binding energies in high-pressure solid hydrogen are smaller than $0.1 \mathrm{eV}$ and cannot be resolved above the statistical and finite-size errors in our DMC results. Many-body perturbation theory calculations of the excitonic gap of the Cmca-12 structure show that the exciton binding energy decreases with increasing pressure from $66 \mathrm{meV}$ at $100 \mathrm{GPa}$ to $12 \mathrm{meV}$ at $200 \mathrm{GPa}$ [54]. Accurate DMC calculations of the exciton binding energy would therefore require an unattainable precision of better than $10 \mathrm{meV}$ in the total energy of the simulation cell. Therefore, in the rest of this paper, we do not attempt to distinguish the excitonic band gap $\Delta_{\text {exc }}$ from the QP band gap $\Delta_{\text {qp }}$.

\section{Backflow}

The simplest possible antisymmetric many-electron trial wave function is a Slater determinant of Hartree-Fock or DFT orbitals. Multiplying the Slater determinant by a Jastrow factor helps to keep electrons away from each other and significantly lowers the energy expectation value calculated in a VMC simulation, but does not change the nodal surface or the fixed-node DMC energy. Introducing a BF transformation [50], which can be viewed as a leading-order improvement to the Slater-Jastrow form [55,56], changes the nodes and thus lowers the DMC energy. Here we have systematically investigated the influence of BF on the fixed-node DMC results for solid hydrogen. We also have addressed the question of how the choice of wave function affects VMC and DMC results.

Band gaps calculated using Hartree-Fock theory, which neglects electron-electron correlation, are generally much too large. DMC calculations using Slater-Jastrow trial wave functions retrieve a high percentage of the correlation energy and produce gaps closer to experimental values. It is unsurprising that improving the DMC description of electronic correlation by adding a $\mathrm{BF}$ transformation further lowers the calculated DMC gap. As shown in Table II, using BF trial wave functions decreases the calculated $\mathrm{QP}$ and excitonic gaps of the $C 2 / c$ structure by $0.5(1)$ and $0.2(1) \mathrm{eV}$, respectively, bringing them within error bars of each other. Although the inclusion of BF considerably improves the DMC results, the computational cost is high. One of the most expensive operations in any DMC code is the evaluation of the orbitals and their first two derivatives, and the evaluation of the collective BF coordinates makes this even slower, because every element of the Slater matrix must be updated every time a single electron is moved. For this reason we did not utilize BF wave functions for the other structures at different pressures.

\section{Finite-size effects in scissors corrections}

Our results indicate that the magnitude of the scissor correction depends on the crystal structure and the applied pressure. To within our statistical error of $0.1(1) \mathrm{eV}$, we found that $\delta_{\text {sci }}(N)$ is independent of system size $N$ for $N \geqslant 200$. The DMC band gap at infinite system size is therefore $\Delta^{\mathrm{DMC}}(N \rightarrow \infty)=\Delta^{\mathrm{DFT}}(N \rightarrow \infty)+\delta_{\text {sci }}$.

The scissor correction $\delta_{\text {sci }}$ has a very weak bond-length dependence, but the DFT band gap $\Delta^{\mathrm{DFT}}$ is sensitive to the molecular bond length. The bond lengths used here lie within $0.0007 \AA$ of those calculated using the van der Waals density functional (vdW-DF) [45], which are known to differ by less than $0.008 \AA$ from the optimized DMC bond lengths for the $C 2 / c$ phase [22]. Figure 6 illustrates the $P 6_{3} / m$ DFT band 

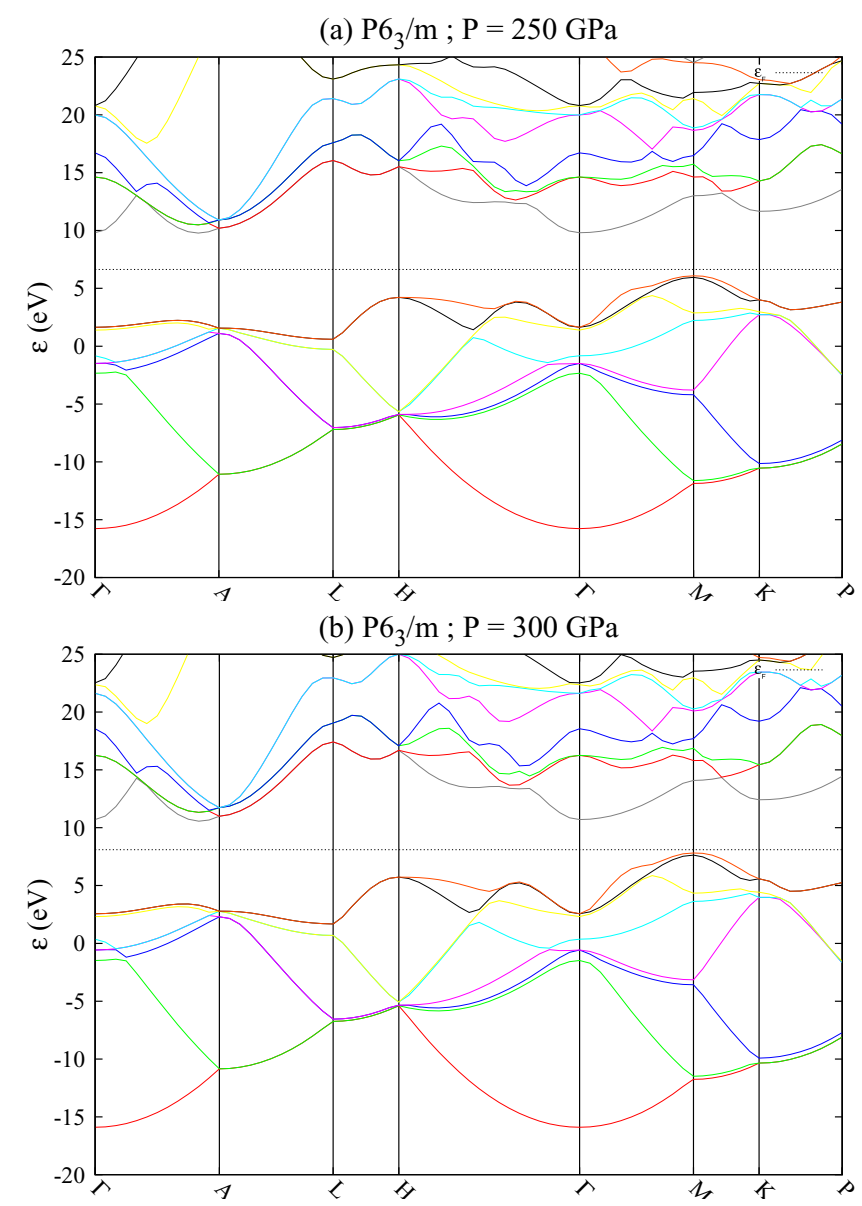

(c) $\mathrm{P}_{3} / \mathrm{m} ; \mathrm{P}=350 \mathrm{GPa}$

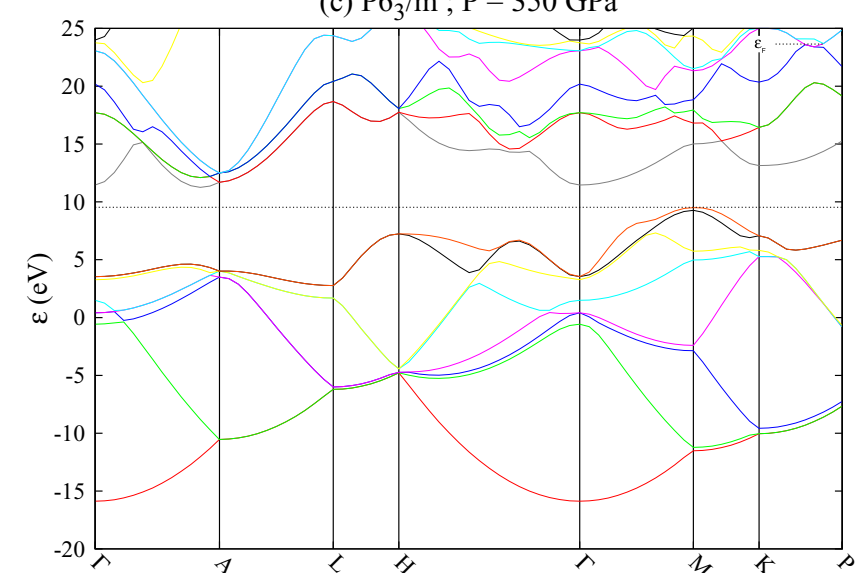

FIG. 5. As Fig. 2, but for the $P 6_{3} / m$ phase at pressures $P=250$, 300 , and $350 \mathrm{GPa}$.

gaps, which were obtained using the BLYP, PBE, and vdW-DF functionals, as a function of $\mathrm{H}-\mathrm{H}$ molecular bond length. The gradient of the band gap with respect to molecular bond length is $\sim 27.3 \mathrm{eV} / \AA$. The same gradient is obtained with each density functional. Multiplying the maximum bond-length error of $0.008+0.0007 \approx 0.009 \AA$ by the gradient of the DFT gap with respect to bond length shows that the resulting error in the static-nucleus gap is no greater than $0.25 \mathrm{eV}$.

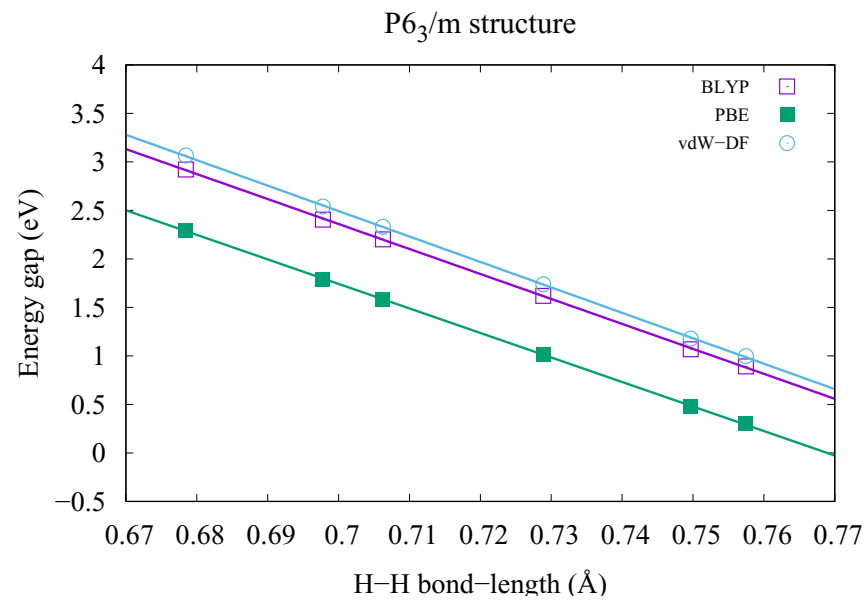

FIG. 6. DFT energy gap as a function of molecular bond length for the $P 6_{3} / \mathrm{m}$ phase at fixed density. The results are calculated using the BLYP, PBE, and vdW-DF functionals.

\section{DMC band gaps}

Table VI shows the static-nucleus DMC band gaps of the $C 2 / c, P c, P b c n$, and $P 6_{3} / m$ structures at pressures of 250 , 300 , and $350 \mathrm{GPa}$. The band gaps of the $C 2 / c$ and $P c$ structures are similar, as are those of the $P b c n$ and $P 6_{3} / m$ structures. The $P 6_{3} / m$ band gaps are slightly greater than those of the other structures studied. A linear extrapolation suggests that the band gaps of the $C 2 / c, P c, P b c n$, and $P 6_{3} / m$ structures vanish at pressures of 464(5), 421(6), 442(5), and 473(4) GPa. DMC calculations of the phase diagram predict that the staticnucleus molecular-to-atomic phase transition also occurs in the pressure range 415-475 $\mathrm{GPa}$ [12].

Figure 7 compares the pressure dependence of the staticnucleus DMC band gaps of the $C 2 / c, P b c n, P c$, and $P 6_{3} / m$ structures of hydrogen with experimental data. The DMC energy gaps of the $P c$ and $C 2 / c$ structures at $300 \mathrm{GPa}$ are close to the absorption-edge measurements for hydrogen at $100 \mathrm{~K}$ and above $300 \mathrm{GPa}$ reported in Ref. [57]. These authors predicted that, at low temperatures, metallic hydrogen should be observed at about $450 \mathrm{GPa}$, when the electronic band gap closes. The $P b c n$ and $P 6_{3} / m$ energy gaps are larger than the experimentally measured gap over the entire pressure range studied. Figure 7 illustrates that there is substantial disagreement between experimental gap measurements.

TABLE VI. DMC band gaps for different high-pressure solid molecular hydrogen structures at pressures of 250, 300, and $350 \mathrm{GPa}$.

\begin{tabular}{lccc}
\hline \hline & \multicolumn{3}{c}{$\Delta^{\mathrm{DMC}}(\mathrm{eV})$} \\
\cline { 2 - 4 } Structure & $250 \mathrm{GPa}$ & $300 \mathrm{GPa}$ & $350 \mathrm{GPa}$ \\
\hline $\mathrm{C} 2 / \mathrm{c}$ & $3.0(2)$ & $2.3(2)$ & $1.6(2)$ \\
$P c$ & $3.2(2)$ & $2.4(2)$ & $1.3(2)$ \\
$P b c n$ & $3.6(2)$ & $2.8(2)$ & $1.7(2)$ \\
$P 6_{3} / m$ & $3.6(2)$ & $2.8(2)$ & $2.0(2)$ \\
\hline \hline
\end{tabular}




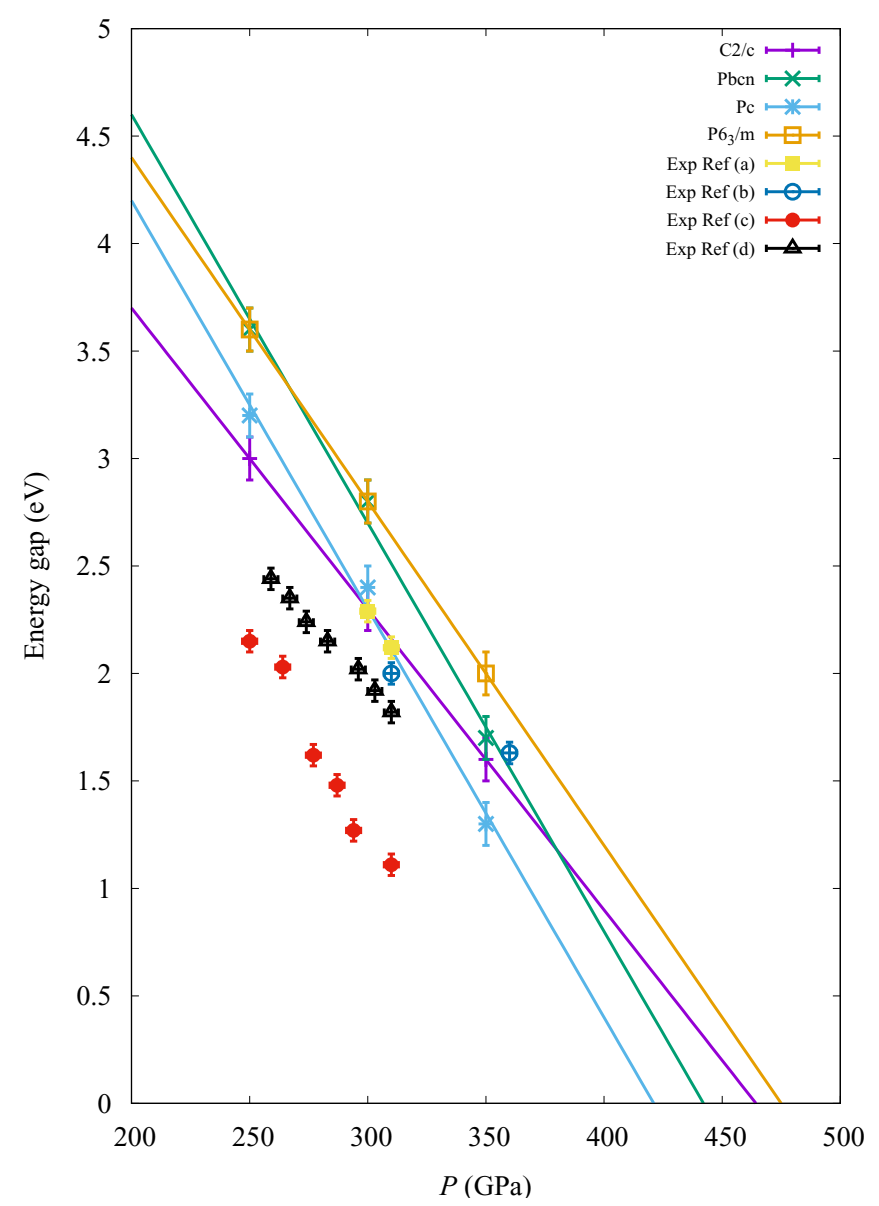

FIG. 7. Static-nucleus DMC energy gaps for the $C 2 / c, P c, P b c n$, and $P 6_{3} / \mathrm{m}$ structures against pressure $P$. References (a) [57], (b) [11], (c) [18], and (d) [7] are energy gaps at different $P( \pm 3 \mathrm{GPa})$ reported by experiments.

\section{B. Nuclear quantum effects}

It is well known [12,34] that nuclear quantum effects (NQEs) are significant in hydrogen-rich systems and affect the phase transitions of high-pressure solid hydrogen. DFT-based path-integral molecular dynamics (PIMD) simulations [21] indicate that the influence of NQEs on the band gap is strongly dependent on the choice of exchange-correlation functional. PIMD results at $T=200 \mathrm{~K}$ obtained using the PBE [58] functional predict that the band gaps of the $C 2 / c$ and $P b c n$ structures close below $250 \mathrm{GPa}$ [21], in disagreement with experiment. PIMD simulations employing the HeydScuseria-Ernzerhof (HSE) [59] functional are not significantly better, although using a van der Waals functional leads to an improvement [21]. These results are surprising because DFT calculations using the hybrid HSE functional normally yield much better ground-state band gaps than calculations using the semilocal PBE functional [60].

Assuming the validity of the Born-Oppenheimer approximation, the full electron-nuclear wave function $\Psi(\mathbf{R}, \mathbf{d})$ may be approximated as $\Phi(\mathbf{R} \mid \mathbf{d}) \chi(\mathbf{d})$, where $\Phi(\mathbf{R} \mid \mathbf{d})$ is a function of the positions $\mathbf{R}=\left(\mathbf{r}_{1}, \mathbf{r}_{2}, \ldots, \mathbf{r}_{N}\right)$ of the $N$ electrons in the supercell at fixed nuclear positions $\mathbf{d}$, and $\chi(\mathbf{d})$ is the nuclear wave function. The band structure as calculated using
PIMD is an average of the band structures corresponding to the electronic wave functions $\Phi(\mathbf{R} \mid \mathbf{d})$, weighted according to the nuclear probability density $|\chi(\mathbf{d})|^{2}$. Since each HSE band gap is likely to be better (wider) than the corresponding PBE band gap, the finite-temperature HSE-PIMD gap ought to be better than the PBE-PIMD gap. The observation that both PIMD gaps are poor suggests, therefore, that both functionals produce inaccurate nuclear probability densities $|\chi(\mathbf{d})|^{2}$. This problem is consistent with other observed failures of DFT for high-pressure hydrogen [25]. Understanding the influence of NQEs and temperature on the band gap of solid hydrogen is a challenging problem that may require going beyond DFT-based methods. We do not address this problem here, but a comparison of our static-nucleus DMC band gaps with experimental results can yield estimates of NQEs.

It is not straightforward to measure the band gap at pressures greater than $300 \mathrm{GPa}$, but the experimental results shown in Fig. 7 suggest that solid hydrogen remains an insulator up to $350 \mathrm{GPa}$ or more. The $C 2 / c$ and $P c$ structures are currently considered [34] the most likely candidates for phases III and IV, respectively, and the $P b c n$ and $P 6_{3} / m$ structures have higher band gaps than these. Despite the inevitable band-gap reduction due to NQEs, we assume that all of the structures considered in this paper have nonzero band gaps at $300 \mathrm{GPa}$ and $300 \mathrm{~K}$. The estimated molecular-to-atomic transition pressure, calculated using static-nucleus DMC calculations together with DFT anharmonic vibrational corrections, is about $374 \mathrm{GPa}$ [12]. According to Fig. 7, the vibrational renormalization of the gap of $C 2 / c$ (the structure believed to correspond to phase III) would have to be $-1.3(2) \mathrm{eV}$ if the gap is to have closed at $374 \mathrm{GPa}$. Assuming that the $P c$ structure is the best candidate for phase IV, as has been reported recently [34], the calculated DMC band gap at $300 \mathrm{GPa}$ is $0.6(2) \mathrm{eV}$ larger than the experimental gap reported in Ref. [7]. The difference is similar to the zero-point renormalization of the diamond band gap at ambient conditions, which was found to be as large as $0.6 \mathrm{eV}$ [61,62], but the atomic mass of carbon is twelve times that of hydrogen and we would expect a larger band-gap reduction here. Other experimental results [18] report an energy gap of $1.2 \mathrm{eV}$ for high pressure hydrogen at $300 \mathrm{~K}$ and pressures around $300 \mathrm{GPa}$. This would imply a NQE band-gap reduction of $1.2(2) \mathrm{eV}$, which we believe to be more plausible. Bearing in mind the expected NQE, our static-nucleus DMC gaps are more consistent with the experimental results reported in Ref. [18] than with those reported in Refs. [57], [11], and [7]. Hence our results suggest that due to the strong zero-point motion and coupling between the band gap and molecular bond length, it is possible all of the graphenelike phases have a significant density of states within the static gap.

The main effect of quantum and thermal vibrations is to increase the intermolecular interactions and weaken the intramolecular bonding. Bearing in mind the symmetries and geometries of the crystals studied, we would expect the NQE-induced band-gap reduction to be larger in the layered $C 2 / c, P c$, and $P b c n$ structures than in the $P 6_{3} / m$ structure. This suggestion is consistent with the high structural flexibility of phase IV observed in ab initio variable-cell molecular dynamics simulations [63] at pressures of 250-350 GPa and temperatures of $300-500 \mathrm{~K}$. Protons in the graphenelike layers 
were seen to transfer readily to neighboring molecular sites via a simultaneous rotation of three-molecule rings. The role played by nuclear dynamics needs to be investigated in subsequent work, and ultimately this prediction about the nature of the phase transition needs to be tested by experiment.

\section{CONCLUSIONS}

In summary, we have performed DMC calculations of the QP and excitonic energy band gaps of solid molecular hydrogen at high pressure. We find that the exciton binding energy is smaller than $100 \mathrm{meV} /$ atom and that our DMC QP and excitonic band gaps are within error bars of one another. We have systematically investigated the energy reductions obtained by introducing a better description of electronic correlation into our VMC and DMC trial wave functions. Using a highly-correlated $\mathrm{BF}$ wave function reduces the DMC band gap and significantly improves the ground-state DMC energy by decreasing the fixed-node errors. A comparison of our DMC band-gap results with experiments suggest that NQEs reduce the band gap significantly, especially in the layered structures. We also find that there is a strong coupling between the band gap and molecular bond length.

\section{ACKNOWLEDGMENTS}

This work was supported by the UK Engineering and Physical Science Research Council under Grant No. EP/K038141/1, by the Thomas Young Centre under Grant No. TYC-101, and by PRACE-3IP project FP7 RI-312763. Computing facilities were provided by ARCHER, the UK National Supercomputing Service, and by the Imperial College London High Performance Computing Centre.
[1] E. Wigner and H. B. Huntington, J. Chem. Phys. 3, 764 (1935).

[2] N. W. Ashcroft, Phys. Rev. Lett. 21, 1748 (1968).

[3] S. A. Bonev, E. Schwegler, T. Ogitsu, and G. Galli, Nature (London) 431, 669 (2004).

[4] H. K. Mao and R. J. Hemley, Rev. Mod. Phys. 66, 671 (1994).

[5] V. L. Ginzburg, Phys. Usp. 42, 353 (1999).

[6] M. I. Eremets and I. A. Troyan, Nat. Mater. 10, 927 (2011).

[7] R. T. Howie, C. L. Guillaume, T. Scheler, A. F. Goncharov, and E. Gregoryanz, Phys. Rev. Lett. 108, 125501 (2012).

[8] R. T. Howie, I. B. Magdău, A. F. Goncharov, G. J. Ackland, and E. Gregoryanz, Phys. Rev. Lett. 113, 175501 (2014).

[9] R. T. Howie, P. Dalladay-Simpson, and E. Gregoryanz, Nat. Mater. 14, 495 (2015).

[10] P. Dalladay-Simpson, R. T. Howie, and E. Gregoryanz, Nature (London) 529, 63 (2016).

[11] C. S. Zha, Z. Liu, and R. J. Hemley, Phys. Rev. Lett. 108, 146402 (2012).

[12] S. Azadi, B. Monserrat, W. M. C. Foulkes, and R. J. Needs, Phys. Rev. Lett. 112, 165501 (2014).

[13] M. Städele and R. M. Martin, Phys. Rev. Lett. 84, 6070 (2000).

[14] K. A. Johnson and N. W. Ashcroft, Nature (London) 403, 632 (2000).

[15] R. P. Dias, O. Noked, and I. F. Silvera, Phys. Rev. Lett. 116, 145501 (2016).

[16] C. J. Pickard and R. J. Needs, Nat. Phys. 3, 473 (2007).

[17] C. J. Pickard, M. Martinez-Canales, and R. J. Needs, Phys. Rev. B 85, 214114 (2012).

[18] A. F. Goncharov, J. S. Tse, H. Wang, J. Yang, V. V. Struzhkin, R. T. Howie, and E. Gregoryanz, Phys. Rev. B 87, 024101 (2013).

[19] I. B. Magdău and G. J. Ackland, Phys. Rev. B 87, 174110 (2013).

[20] I. I. Naumov, R. E. Cohen, and R. J. Hemley, Phys. Rev. B 88, 045125 (2013).

[21] M. A. Morales, J. M. McMahon, C. Pierleoni, and D. M. Ceperley, Phys. Rev. B 87, 184107 (2013).

[22] R. C. Clay, J. Mcminis, J. M. McMahon, C. Pierleoni, D. M. Ceperley, and M. A. Morales, Phys. Rev. B 89, 184106 (2014).

[23] S. Azadi and T. D. Kühne, JETP Lett. 95, 449 (2012).
[24] R. Singh, S. Azadi, and T. D. Kühne, Phys. Rev. B 90, 014110 (2014).

[25] S. Azadi and W. M. C. Foulkes, Phys. Rev. B 88, 014115 (2013).

[26] S. Azadi, W. M. C. Foulkes, and T. D. Kühne, New J. Phys. 15, 113005 (2013).

[27] J. P. Perdew, A. Ruzsinszky, L. A. Constantin, J. Sun, and G. I. Csonka, J. Chem. Theory Comput. 5, 902 (2009).

[28] W. M. C. Foulkes, L. Mitas, R. J. Needs, and G. Rajagopal, Rev. Mod. Phys. 73, 33 (2001).

[29] E. Mostaani, N. D. Drummond, and V. I. Fal'ko, Phys. Rev. Lett. 115, 115501 (2015).

[30] J. Kolorenč and L. Mitas, Rep. Prog. Phys. 74, 026502 (2011).

[31] S. Azadi and W. M. C. Foulkes, J. Chem. Phys. 143, 102807 (2015).

[32] S. Azadi and R. E. Cohen, J. Chem. Phys. 143, 104301 (2015).

[33] S. Azadi and R. E. Cohen, J. Chem. Phys. 145, 064501 (2016).

[34] N. D. Drummond, B. Monserrat, J. H. Lloyd-Williams, P. López Ríos, C. J. Pickard, and R. J. Needs, Nat. Commun. 6, 7794 (2015).

[35] L. Mitas and R. M. Martin, Phys. Rev. Lett. 72, 2438 (1994).

[36] A. J. Williamson, R. Q. Hood, R. J. Needs, and G. Rajagopal, Phys. Rev. B 57, 12140 (1998).

[37] M. D. Towler, R. Q. Hood, and R. J. Needs, Phys. Rev. B 62, 2330 (2000).

[38] N. D. Drummond, R. J. Needs, A. Sorouri, and W. M. C. Foulkes, Phys. Rev. B 78, 125106 (2008).

[39] J. McMinis, R. C. Clay, D. Lee, and M. A. Morales, Phys. Rev. Lett. 114, 105305 (2015).

[40] J. M. McMahon, M. A. Morales, C. Pierleoni, and D. M. Ceperley, Rev. Mod. Phys. 84, 1607 (2012).

[41] P. Giannozzi, S. Baroni, N. Bonini, M. Calandra, R. Car, C. Cavazzoni, D. Ceresoli, G. L. Chiarotti, M. Cococcioni, I. Dabo, A. Dal Corso, S. de Gironcoli, S. Fabris, G. Fratesi, R. Gebauer, U. Gerstmann, C. Gougoussis, A. Kokalj, M. Lazzeri, L. Martin-Samos, N. Marzari, F. Mauri, R. Mazzarello, S. Paolini, A. Pasquarello, L. Paulatto, C. Sbraccia, S. Scandolo, G. Sclauzero, A. P. Seitsonen, A. Smogunov, P. Umari, and R. M. Wentzcovitch, J. Phys.: Condens. Matter 21, 395502 (2009). 
[42] S. J. Clark, M. D. Segall, C. J. Pickard, P. J. Hasnip, M. J. Probert, K. Refson, and M. C. Payne, Z. Kristallogr. 220, 567 (2005).

[43] C. Lee, W. Yang, and R. G. Parr, Phys. Rev. B 37, 785 (1988).

[44] See Supplemental Material at http://link.aps.org/supplemental/ 10.1103/PhysRevB.95.035142 for DFT optimized geometries of all the studied phases at pressures of 250, 300, and $350 \mathrm{GPa}$.

[45] M. Dion, H. Rydberg, E. Schröder, D. C. Langreth, and B. I. Lundqvist, Phys. Rev. Lett. 92, 246401 (2004).

[46] R. J. Needs, M. D. Towler, N. D. Drummond, and P. López Ríos, J. Phys.: Condens. Matter 22, 023201 (2010).

[47] S. Azadi, C. Cavazzoni, and S. Sorella, Phys. Rev. B 82, 125112 (2010).

[48] D. Alfè and M. J. Gillan, Phys. Rev. B 70, 161101(R) (2004).

[49] N. D. Drummond, M. D. Towler, and R. J. Needs, Phys. Rev. B 70, 235119 (2004).

[50] P. López Ríos, A. Ma, N. D. Drummond, M. D. Towler, and R. J. Needs, Phys. Rev. E 74, 066701 (2006).

[51] C. J. Umrigar, K. G. Wilson, and J. W. Wilkins, Phys. Rev. Lett. 60, 1719 (1988)
[52] N. D. Drummond and R. J. Needs, Phys. Rev. B 72, 085124 (2005).

[53] E. Mostaani, B. Monserrat, N. D. Drummond, and C. J. Lambert, Phys. Chem. Chem. Phys. 18, 14810 (2016).

[54] M. Dvorak, X-J. Chen, and Z. Wu, Phys. Rev. B 90, 035103 (2014).

[55] M. Holzmann, D. M. Ceperley, C. Pierleoni, and K. Esler, Phys. Rev. E 68, 046707 (2003).

[56] Y. Kwon, D. M. Ceperley, and R. M. Martin, Phys. Rev. B 48, 12037 (1993).

[57] P. Loubeyre, F. Occelli, and R. LeToullec, Nature (London) 416, 613 (2002).

[58] J. P. Perdew, K. Burke, and M. Ernzerhof, Phys. Rev. Lett. 77, 3865 (1996).

[59] J. Heyd, G. E. Scuseria, and M. Ernzerhof, J. Chem. Phys. 118, 8207 (2003).

[60] J. Paier, M. Marsman, K. Hummer, G. Kresse, I. C. Gerber, and J. G. Ángyán, J. Chem. Phys. 124, 154709 (2006).

[61] F. Giustino, S. G. Louie, and M. L. Cohen, Phys. Rev. Lett. 105, 265501 (2010).

[62] A. Marini, Phys. Rev. Lett. 101, 106405 (2008).

[63] H. Liu and Y. Ma, Phys. Rev. Lett. 110, 025903 (2013). 